\title{
Common Coupled Fixed Points for Four Maps Using Property(E.A.) in Complex Valued $b$ - Metric Spaces
}

\author{
K.P.R.Rao ${ }^{1 *}$, Sk.Sadik ${ }^{2}$ and S.V.K.Sarma ${ }^{3}$ \\ ${ }^{1}$ Department of Mathematics, Acharya Nagarjuna University, Nagarjuna Nagar -522 510, A.P., India. \\ ${ }^{2}$ Department of Mathematics, Sir C R R College of Engineering, Eluru, West Godhawari - 534007, Andhra \\ Pradesh, India \\ ${ }^{3}$ Department of S and H(Mathematics), Dhanekula Institute of Engineering and Technology, Ganguru, \\ Penamaluru Mandalam, Vijayawada -521139 \\ Email: kprrao2004@yahoo.com
}

\begin{abstract}
In this paper, we obtain a unique common coupled fixed point theorem for four self maps using property(E.A.)in complex valued $b$-metric spaces. Also we give an example to illustrate our main theorem.
\end{abstract}

Keywords: Complete metric spaces, property(E.A), w-compatible mappings

\section{Introduction and Preliminaries}

It is a well-known fact that the mathematical results regarding fixed points of contraction type mappings are very useful for determining the existence and uniqeness of solutions to various mathematical models.

Azam et al.[1] introduced the notion of a complex valued metric space which is a generalization of the classical metric space and obtained sufficent conditions for the existence of common fixed points of a pair of mappings satisfying a rational contractive condition.Later several authors proved fixed and common fixed point theorems in complex valued metric spaces ,for example, refer to $[2,3,4,6,9,10,13,15,16,18,25]$. Recently some authors,for example[5,12,14,19,21,22,23],obtained coupled and common coupled fixed point theorems for a pair of mappings in complex valued metric spaces.

In this paper,we prove a unique common coupled fixed point theorem for two pairs of mappings satisfying a contractive condition of rational type in the frame work of complex valued $b$-metric spaces using property(E.A.).

To begin, we recall some basic definitions, notations and results.

Throughout this paper $\mathcal{R}, \mathcal{R}^{+}, \mathcal{N}$ and $\mathbb{C}$ denote the set of all real numbers, non-negative real numbers, positive integers and complex numbers respectively.First we refer to the following preliminaries.

Let $z_{1}, z_{2} \in \mathbb{C}$. Define a partial order $\precsim$ on $\mathbb{C}$ as follows: $z_{1} \precsim z_{2}$ if and only if $\operatorname{Re}\left(z_{1}\right) \leq \operatorname{Re}\left(z_{2}\right), \operatorname{Im}\left(z_{1}\right) \leq$ $\operatorname{Im}\left(z_{2}\right)$. Thus $z_{1} \precsim z_{2}$ if one of the following holds:

$$
\begin{aligned}
& (1) \operatorname{Re}\left(z_{1}\right)=\operatorname{Re}\left(z_{2}\right) \text { and } \operatorname{Im}\left(z_{1}\right)=\operatorname{Im}\left(z_{2}\right), \\
& (2) \operatorname{Re}\left(z_{1}\right)<\operatorname{Re}\left(z_{2}\right) \text { and } \operatorname{Im}\left(z_{1}\right)=\operatorname{Im}\left(z_{2}\right), \\
& (3) \operatorname{Re}\left(z_{1}\right)=\operatorname{Re}\left(z_{2}\right) \text { and } \operatorname{Im}\left(z_{1}\right)<\operatorname{Im}\left(z_{2}\right), \\
& (4) \operatorname{Re}\left(z_{1}\right)<\operatorname{Re}\left(z_{2}\right) \text { and } \operatorname{Im}\left(z_{1}\right)<\operatorname{Im}\left(z_{2}\right) .
\end{aligned}
$$

Definition 1.1 ([1]) Let $X$ be a non empty set. A function $d: X \times X \rightarrow \mathbb{C}$ is called a complex valued metric on $X$ if for all $x, y, z \in X$ the following conditions are satisfied:

(i) $0 \precsim d(x, y)$ and $d(x, y)=0$ if and only if $x=y$;

(ii) $d(x, y)=d(y, x)$;

(iii) $d(x, y) \precsim d(x, z)+d(z, y)$.

The pair $(X, d)$ is called a complex valued metric space.

Now, we briefly review the notation about complex valued b-metric spaces. 
Definition 1.2 ([7]) Let $X$ be a non empty set and $s \geq 1$. A function $d: X \times X \rightarrow \mathbb{C}$ is called a complex valued $b$ - metric on $X$ if for all $x, y, z \in X$ the following conditions are satisfied:

(i) $0 \precsim d(x, y)$ and $d(x, y)=0$ if and only if $x=y$;

(ii) $d(x, y)=d(y, x)$;

(iii) $d(x, y) \precsim s[d(x, z)+d(z, y)]$.

The pair $(X, d)$ is called a complex valued $b$ - metric space.

Remark 1.3 Let $(X, d)$ be a complex valued $b$-metric space with $s \geq 1$. Then

(i) $|d(x, y)|$ or $|d(u, v)|<|1+s d(x, y)+s d(u, v)|, \forall x, y, u, v \in X$.

(ii) If $x \neq y$ then $|d(x, y)|>0$.

(iii) For $0 \leq k<1$ and $z, w \in \mathbb{C}$, if $|z| \leq k|w|$ and $|w| \leq k|z|$ then $z=w=0$.

Definition $1.4([7])$ Let $(X, d)$ be a complex valued b-metric space.

(i) A point $x \in X$ is called an interior point of a set $A \subseteq X$ whenever there exists $0 \prec r \in \mathbb{C}$ such that $B(x, r)=\{y \in X: d(x, y) \prec r\} \subseteq A$.

(ii) A point $x \in X$ is called a limit point of a set $A \subseteq X$ whenever there exists $0 \prec r \in \mathbb{C}$ such that $B(x, r) \cap(X-A) \neq \phi$.

(iii) $A$ subset $B \subseteq X$ is called open whenever each point of $B$ is an interior point of $B$.

(iv) $A$ subset $B \subseteq X$ is called closed whenever each limit point of $B$ is in $B$.

(v) The family $F=\{B(x, r): x \in X$ and $0 \prec r\}$ is a sub basis for a topology on $X$. We denote this complex topology by $\tau_{c}$. Indeed, the topology $\tau_{c}$ is Hausdorff.

Let $\left\{x_{n}\right\}$ be a sequence in $X$ and $x \in X$.If for every $c \in \mathbb{C}$ with $0 \preceq c$ there is $n_{0} \in \mathcal{N}$ such that for all $n>n_{0}, d\left(x_{n}, x\right) \prec c$, then $\left\{x_{n}\right\}$ is said to be convergent to $x$ and $x$ is the limit point of $\left\{x_{n}\right\}$. We denote this by $\lim _{n \rightarrow \infty} x_{n}=x$ or $x_{n} \rightarrow x$ as $n \rightarrow \infty$. If for every $c \in \mathbb{C}$ with $0 \prec c$ there is $n_{0} \in \mathcal{N}$ such that for all $n>n_{0}, d\left(x_{n}, x_{n+m}\right) \prec c$, where $m \in \mathcal{N}$, then $\left\{x_{n}\right\}$ is called a Cauchy sequence in $(X, d)$. If every Cauchy sequence is convergent in $(X, d)$ then $(X, d)$ is called a complete complex valued $b$-metric space. We require the following lemmas.

Lemma 1.5 ([r]) Let $(X, d)$ be a complex valued b-metric space and let $\left\{x_{n}\right\}$ be a sequence in $X$.Then $\left\{x_{n}\right\}$ converges to $x$ if and only if $\left|d\left(x_{n}, x\right)\right| \rightarrow 0$ as $n \rightarrow \infty$.

Lemma 1.6 ([r]) Let $(X, d)$ be a complex valued b-metric space and let $\left\{x_{n}\right\}$ be a sequence in $X$.Then $\left\{x_{n}\right\}$ is a Cauchy sequence if and only if $\left|d\left(x_{n}, x_{n+m}\right)\right| \rightarrow 0$ as $n, m \rightarrow \infty$.

One can easily prove the following lemma

Lemma 1.7 Let $(X, d)$ be a complex valued b-metric space and let $\left\{x_{n}\right\}$ and $\left\{y_{n}\right\}$ be sequences in $X$ converging to $x$ and $y$ respectively. Then

(i) $\frac{1}{s}|d(x, z)| \leq \lim _{n \rightarrow \infty}\left|d\left(x_{n}, z\right)\right| \leq s|d(x, z)|$ for all $z \in X$,

(ii) $\frac{1}{s^{2}}|d(x, y)| \leq \lim _{n \rightarrow \infty}\left|d\left(x_{n}, y_{n}\right)\right| \leq s^{2}|d(x, y)|$.

Recently Bhaskar and Lakshmikantham [20] introduced the concept of coupled fixed point and discussed some problems of the uniqueness of a coupled fixed point and applied their results to the problems of the existence and uniqueness of a solution for the periodic boundary value problems. Later Lakshmikantham and Ciric [24] proved some coupled coincidence and coupled common fixed point results in partially ordered metric spaces.

Definition 1.8 Let $X$ be a non empty set and $F: X \times X \rightarrow X$ and $S: X \rightarrow X$.

(i)([24]) An element $(x, y) \in X \times X$ is called a common coupled fixed point of mappings $F$ and $S$ if $x=S x=F(x, y)$ and $y=S y=F(y, x)$.

(ii)([8]) The pair $(F, S)$ is called $w$-compatible if $S(F(x, y))=F(S x, S y)$ and $S(F(y, x))=F(S y, S x)$, whenever $S x=F(x, y)$ and $S y=F(y, x)$. 
Verma and Pathak [17]defined property(E.A.),like[11]in complex valued metric spaces as follows.

Definition 1.9 ([17]) Let $A, S: X \rightarrow X$ be two self-maps of a complex-valued metric space $(X, d)$. The pair $(A, S)$ is said to satisfy property (E.A), if there exists a sequence $\left\{x_{n}\right\}$ in $X$ such that $\lim _{n \rightarrow \infty} A x_{n}=$ $\lim _{n \rightarrow \infty} S x_{n}=t$ for some $t \in X$.

In this paper, we define property(E.A.) for two mappings $F: X \times X \rightarrow X$ and $S: X \rightarrow X$ as follows in complex valued metric spaces.

Definition 1.10 Let $X$ be a nonempty set and $F: X \times X \rightarrow X$ and $S: X \rightarrow X$ be two mappings. Then the pair $(F, S)$ is said to satisfy property(E.A.) if there exist sequences $\left\{x_{n}\right\}$ and $\left\{y_{n}\right\}$ in $X$ such that for some $t, t^{\prime} \in X$, we have $\lim _{n \rightarrow \infty} F\left(x_{n}, y_{n}\right)=\lim _{n \rightarrow \infty} S x_{n}=t$ and $\lim _{n \rightarrow \infty} F\left(y_{n}, x_{n}\right)=\lim _{n \rightarrow \infty} S y_{n}=t^{\prime}$.

\section{Main Result}

Theorem 2.1 Let $(X, d)$ be a complex valued b-metric space with $s \geq 1$. Let $F, G: X \times X \rightarrow X$ and $S, T: X \rightarrow X$ be mappings satisfying the following :

$$
\begin{aligned}
& s^{3} d(F(x, y), G(u, v)) \\
& \precsim a_{1} d(S x, T u)+a_{2} d(S y, T v)+a_{3} d(S x, F(x, y)) \\
& \quad+a_{4} d(S y, F(y, x))+a_{5} d(T u, G(u, v))+a_{6} d(T v, G(v, u)) \\
& \quad+a_{7} d(S x, G(u, v))+a_{8} d(S y, G(v, u))+a_{9} d(T u, F(x, y))+a_{10} d(T v, F(y, x)) \\
& \quad+a_{11} \frac{d(S x, F(x, y)) d(T u, G(u, v))}{1+s d(S x, T u)+s d(S y, T v)}+a_{12} \frac{d(T u, F(x, y)) d(S x, G(u, v))}{1+s d(S x, T u)+s d(S y, T v)}
\end{aligned}
$$

for all $x, y, u, v \in X$, where $a_{i}, i=1,2, \ldots ., 12$ are non-negative real numbers such that $\sum_{i=1}^{12} a_{i}<\frac{1}{s}$.

(2.1.2) The pairs $(F, S)$ and $(G, T)$ are $w$-compatible.

(2.1.3)(a) The pair $(F, S)$ satisfies property(E.A.), $F(X \times X) \subseteq T(X)$ and $S(X)$ is a closed sub space of $X$.

$$
\text { (or) }
$$

$(2.1 .3)(b)$ The pair $(G, T)$ satisfies property(E.A.), $G(X \times X) \subseteq S(X)$ and $T(X)$ is a closed sub space of $X$.

Then $F, G, S$ and $T$ have a unique common coupled fixed point.

Proof. Suppose (2.1.3)(a) holds.

Then there exist sequences $\left\{x_{n}\right\}$ and $\left\{y_{n}\right\}$ in $X$ such that $\lim _{n \rightarrow \infty} F\left(x_{n}, y_{n}\right)=\lim _{n \rightarrow \infty} S x_{n}=t$ and $\lim _{n \rightarrow \infty} F\left(y_{n}, x_{n}\right)=\lim _{n \rightarrow \infty} S y_{n}=t^{\prime}$ for some $t$ and $t^{\prime}$ in $X$.

Since $F(X \times X) \stackrel{n \rightarrow \infty}{\subseteq} T(X)$, there exist sequences $\left\{z_{n}\right\}$ and $\left\{w_{n}\right\}$ in $X$ such that $F\left(x_{n}, y_{n}\right)=T z_{n} \rightarrow t$ and $F\left(y_{n}, x_{n}\right)=T w_{n} \rightarrow t^{\prime}$ as $n \rightarrow \infty$.

Consider

$$
\begin{aligned}
& s\left|d\left(t, G\left(z_{n}, w_{n}\right)\right)\right| \\
& \leq s^{2}\left|d\left(t, F\left(x_{n}, y_{n}\right)\right)\right|+s^{2}\left|d\left(F\left(x_{n}, y_{n}\right), G\left(z_{n}, w_{n}\right)\right)\right| \\
& \leq s^{2}\left|d\left(t, F\left(x_{n}, y_{n}\right)\right)\right|+s^{3}\left|d\left(F\left(x_{n}, y_{n}\right), G\left(z_{n}, w_{n}\right)\right)\right|, \text { since } s \geq 1 \\
& \leq s^{2}\left|d\left(t, F\left(x_{n}, y_{n}\right)\right)\right|+a_{1}\left|d\left(S x_{n}, T z_{n}\right)\right|+a_{2}\left|d\left(S y_{n}, T w_{n}\right)\right| \\
& \quad+a_{3}\left|d\left(S x_{n}, F\left(x_{n}, y_{n}\right)\right)\right|+a_{4}\left|d\left(S y_{n}, F\left(y_{n}, x_{n}\right)\right)\right|+a_{5}\left|d\left(T z_{n}, G\left(z_{n}, w_{n}\right)\right)\right| \\
& \quad+a_{6}\left|d\left(T w_{n}, G\left(w_{n}, z_{n}\right)\right)\right|+a_{7}\left|d\left(S x_{n}, G\left(z_{n}, w_{n}\right)\right)\right|+a_{8}\left|d\left(S y_{n}, G\left(w_{n}, z_{n}\right)\right)\right| \\
& \quad+a_{9}\left|d\left(T z_{n}, F\left(x_{n}, y_{n}\right)\right)\right|+a_{10}\left|d\left(T w_{n}, F\left(y_{n}, x_{n}\right)\right)\right| \\
& \quad+a_{11} \frac{\left|d\left(S x_{n}, F\left(x_{n}, y_{n}\right)\right)\right|\left|d\left(T z_{n}, G\left(z_{n}, w_{n}\right)\right)\right|}{\left|1+s d\left(S x_{n}, T z_{n}\right)+s d\left(S y_{n}, T w_{n}\right)\right|} \\
& \quad+a_{12} \frac{\left|d\left(S x_{n}, G\left(z_{n}, w_{n}\right)\right)\right|\left|d\left(T z_{n}, F\left(x_{n}, y_{n}\right)\right)\right|}{\left|1+s d\left(S x_{n}, T z_{n}\right)+s d\left(S y_{n}, T w_{n}\right)\right|} .
\end{aligned}
$$


Letting $n \rightarrow \infty$ and using Lemma 1.7, we get

$$
\begin{aligned}
& s \lim _{n \rightarrow \infty}\left|d\left(t, G\left(z_{n}, w_{n}\right)\right)\right| \leq s^{2}(0)+a_{1}(0)+a_{2}(0)+a_{3}(0)+a_{4}(0)+a_{5} s \lim _{n \rightarrow \infty}\left|d\left(t, G\left(z_{n}, w_{n}\right)\right)\right| \\
& +a_{6} s \lim _{n \rightarrow \infty}\left|d\left(t^{\prime}, G\left(w_{n}, z_{n}\right)\right)\right|+a_{7} s \lim _{n \rightarrow \infty}\left|d\left(t, G\left(z_{n}, w_{n}\right)\right)\right| \\
& +a_{8} s \lim _{n \rightarrow \infty}\left|d\left(t^{\prime}, G\left(w_{n}, z_{n}\right)\right)\right|+a_{9}(0)+a_{10}(0)+a_{11}(0)+a_{12}(0) \\
& =\left(a_{5}+a_{7}\right) s \lim _{n \rightarrow \infty}\left|d\left(t, G\left(z_{n}, w_{n}\right)\right)\right|+\left(a_{6}+a_{8}\right) s \lim _{n \rightarrow \infty}\left|d\left(t^{\prime}, G\left(w_{n}, z_{n}\right)\right)\right|
\end{aligned}
$$

Thus

$$
\lim _{n \rightarrow \infty}\left|d\left(t, G\left(z_{n}, w_{n}\right)\right)\right| \leq \frac{a_{6}+a_{8}}{1-a_{5}-a_{7}} \lim _{n \rightarrow \infty}\left|d\left(t^{\prime}, G\left(w_{n}, z_{n}\right)\right)\right| .
$$

Similarly, we can show that

$$
\lim _{n \rightarrow \infty}\left|d\left(t^{\prime}, G\left(w_{n}, z_{n}\right)\right)\right| \leq \frac{a_{6}+a_{8}}{1-a_{5}-a_{7}} \lim _{n \rightarrow \infty}\left|d\left(t, G\left(z_{n}, w_{n}\right)\right)\right| .
$$

Hence $\lim _{n \rightarrow \infty}\left|d\left(t, G\left(z_{n}, w_{n}\right)\right)\right|=0=\lim _{n \rightarrow \infty}\left|d\left(t^{\prime}, G\left(w_{n}, z_{n}\right)\right)\right|$.

Thus $G\left(z_{n}, w_{n}\right) \rightarrow t$ and $G\left(w_{n}, z_{n}\right) \rightarrow t^{\prime}$, by Lemma 1.5 .

Since $S(X)$ is a closed sub space of $X$, there exist $u, v \in X$ such that

$\lim _{n \rightarrow \infty} S x_{n}=t=S u$ and $\lim _{n \rightarrow \infty} S y_{n}=t^{\prime}=S v$.

Using (2.1.1) and Lemma 1.7, we have

$$
\begin{aligned}
& s|d(F(u, v), S u)|=s^{2} \frac{1}{s}|d(F(u, v), S u)| \\
& \leq s^{3} \lim _{n \rightarrow \infty}\left|d\left(F(u, v), G\left(z_{n}, w_{n}\right)\right)\right|, \text { since } s \geq 1 \\
& \leq \lim _{n \rightarrow \infty}\left\{\begin{array}{l}
a_{1}\left|d\left(S u, T z_{n}\right)\right|+a_{2}\left|d\left(S v, T w_{n}\right)\right|+a_{3}|d(S u, F(u, v))|+a_{4}|d(S v, F(v, u))| \\
+a_{5}\left|d\left(T z_{n}, G\left(z_{n}, w_{n}\right)\right)\right|+a_{6}\left|d\left(T w_{n}, G\left(w_{n}, z_{n}\right)\right)\right|+a_{7}\left|d\left(S u, G\left(z_{n}, w_{n}\right)\right)\right| \\
+a_{8}\left|d\left(S v, G\left(w_{n}, z_{n}\right)\right)\right|+a_{9}\left|d\left(T z_{n}, F(u, v)\right)\right|+a_{10}\left|d\left(T w_{n}, F(v, u)\right)\right| \\
+a_{11} \frac{|d(S u, F(u, v))|\left|d\left(T z_{n}, G\left(z_{n}, w_{n}\right)\right)\right|}{\left|1+s d\left(S u, T z_{n}\right)+s d\left(S v, T w_{n}\right)\right|}+a_{12} \frac{\left|d\left(S u, G\left(z_{n}, w_{n}\right)\right)\right|\left|d\left(T z_{n}, F(u, v)\right)\right|}{\left|1+s d\left(S u, T z_{n}\right)+s d\left(S v, T w_{n}\right)\right|}
\end{array}\right\} \\
& \leq a_{3}|d(S u, F(u, v))|+a_{4}|d(S v, F(v, u))|+a_{9} s|d(S u, F(u, v))|+a_{10} s|d(S v, F(v, u))| \\
& \leq s\left(a_{3}+a_{9}\right)|d(S u, F(u, v))|+s\left(a_{4}+a_{10}\right)|d(S v, F(v, u))|, \operatorname{since} s \geq 1
\end{aligned}
$$

Thus $|d(F(u, v), S u)| \leq \frac{\left(a_{4}+a_{10}\right)}{1-a_{3}-a_{9}}|d(S v, F(v, u))|$.

Similarly, we can show that $|d(F(v, u), S v)| \leq \frac{\left(a_{4}+a_{10}\right)}{1-a_{3}-a_{9}}|d(S u, F(u, v))|$. Hence $F(u, v)=S u=t$ and $F(v, u)=S v=t^{\prime}$

Since $(F, S)$ is $w$-compatible, we have $F\left(t, t^{\prime}\right)=S t$ and $F\left(t^{\prime}, t\right)=S t^{\prime}$.

Since $F(X \times X) \subseteq T(X)$, there exist $\alpha, \beta \in X$ such that $t=F(u, v)=T \alpha$ and $t^{\prime}=F(v, u)=T \beta$.

$$
\begin{aligned}
& d(t, G(\alpha, \beta)) \leq s^{3} d(F(u, v), G(\alpha, \beta)) \\
& \leq a_{1} d(S u, T \alpha)+a_{2} d(S v, T \beta)+a_{3} d(S u, F(u, v))+a_{4} d(S v, F(v, u)) \\
& \quad+a_{5} d(T \alpha, G(\alpha, \beta))+a_{6} d(T \beta, G(\beta, \alpha))+a_{7} d(S u, G(\alpha, \beta)) \\
& \quad+a_{8} d(S v, G(\beta, \alpha))+a_{9} d(T \alpha, F(u, v))+a_{10} d(T \beta, F(v, u)) \\
& \quad+a_{11} \frac{d(S u, F(u, v)) d(T \alpha, G(\alpha, \beta))}{1+s d(S u, T \alpha)+s d(S v, T \beta)}+a_{12} \frac{d(T \alpha, F(u, v)) d(S u, G(\alpha, \beta))}{1+s d(S u, T \alpha)+s d(S v, T \beta)} \\
& =a_{5} d(t, G(\alpha, \beta))+a_{6} d\left(t^{\prime}, G(\beta, \alpha)\right)+a_{7} d(t, G(\alpha, \beta))+a_{8} d\left(t^{\prime}, G(\beta, \alpha)\right) \\
& =\left(a_{5}+a_{7}\right) d(t, G(\alpha, \beta))+\left(a_{6}+a_{8}\right) d\left(t^{\prime}, G(\beta, \alpha)\right) .
\end{aligned}
$$

Thus $|d(t, G(\alpha, \beta))| \leq \frac{a_{6}+a_{8}}{1-a_{5}-a_{7}}\left|d\left(t^{\prime}, G(\beta, \alpha)\right)\right|$.

Similarly, we can show that $\left|d\left(t^{\prime}, G(\beta, \alpha)\right)\right| \leq \frac{a_{6}+a_{8}}{1-a_{5}-a_{7}}|d(t, G(\alpha, \beta))|$.

Hence $G(\alpha, \beta)=t=T \alpha$ and $G(\beta, \alpha)=t^{\prime}=T \beta$.

Since $(G, T)$ is $w$-compatible, we have $G\left(t, t^{\prime}\right)=T t$ and $G\left(t^{\prime}, t\right)=T t^{\prime}$

Consider

$$
\begin{aligned}
& 1+d(S t, t)+d\left(S t^{\prime}, t^{\prime}\right) \\
& \precsim 1+s d\left(S t, T z_{n}\right)+s d\left(T z_{n}, t\right)+s d\left(S t^{\prime}, T w_{n}\right)+s d\left(T w_{n}, t^{\prime}\right) \\
& \left|1+d(S t, t)+d\left(S t^{\prime}, t^{\prime}\right)\right| \\
\leq & \left|1+s d\left(S t, T z_{n}\right)+s d\left(S t^{\prime}, T w_{n}\right)\right|+s\left|d\left(T z_{n}, t\right)\right|+s\left|d\left(T w_{n}, t^{\prime}\right)\right| .
\end{aligned}
$$


Letting $n \rightarrow \infty$ and using Lemma 1.5, we get

$$
\left|1+d(S t, t)+d\left(S t^{\prime}, t^{\prime}\right)\right| \leq \lim _{n \rightarrow \infty}\left|1+s d\left(S t, T z_{n}\right)+s d\left(S t^{\prime}, T w_{n}\right)\right|
$$

From(2.1.1) and using Lemma 1.7, we get

$$
\begin{aligned}
& s^{2}|d(S t, t)|=s^{3} \frac{1}{s}\left|d\left(F\left(t, t^{\prime}\right), t\right)\right| \leq s^{3} \lim _{n \rightarrow \infty}\left|d\left(F\left(t, t^{\prime}\right), G\left(z_{n}, w_{n}\right)\right)\right| \\
& \leq \lim _{n \rightarrow \infty}\left[\begin{array}{l}
a_{1}\left|d\left(S t, T z_{n}\right)\right|+a_{2}\left|d\left(S t^{\prime}, T w_{n}\right)\right|+a_{3}\left|d\left(S t, F\left(t, t^{\prime}\right)\right)\right|+a_{4}\left|d\left(S t^{\prime}, F\left(t^{\prime}, t\right)\right)\right| \\
+a_{5}\left|d\left(T z_{n}, G\left(z_{n}, w_{n}\right)\right)\right|+a_{6}\left|d\left(T w_{n}, G\left(w_{n}, z_{n}\right)\right)\right|+a_{7}\left|d\left(S t, G\left(z_{n}, w_{n}\right)\right)\right| \\
+a_{8}\left|d\left(S t^{\prime}, G\left(w_{n}, z_{n}\right)\right)\right|+a_{9}\left|d\left(T z_{n}, F\left(t, t^{\prime}\right)\right)\right|+a_{10}\left|d\left(T w_{n}, F\left(t^{\prime}, t\right)\right)\right|+ \\
a_{11} \frac{\left|d\left(S t, F\left(t, t^{\prime}\right)\right)\right|\left|d\left(T z_{n}, G\left(z_{n}, w_{n}\right)\right)\right|}{\left|1+s d\left(S t, T z_{n}\right)+s d\left(S t^{\prime}, T w_{n}\right)\right|}+a_{12} \frac{\left.\mid d\left(S t, G\left(z_{n}, w_{n}\right)\right)\right)|| d\left(T z_{n}, F\left(t, t^{\prime}\right)\right) \mid}{\left|1+s d\left(S t, T z_{n}\right)+s d\left(S t^{\prime}, T w_{n}\right)\right|}
\end{array}\right] \\
& \leq a_{1} s|d(S t, t)|+a_{2} s\left|d\left(S t^{\prime}, t^{\prime}\right)\right|+a_{7} s|d(S t, t)|+a_{8} s\left|d\left(S t^{\prime}, t^{\prime}\right)\right|+a_{9} s|d(S t, t)| \\
& +a_{10} s\left|d\left(S t^{\prime}, t^{\prime}\right)\right|+a_{12} \frac{s|d(S t, t)| s|d(S t, t)|}{\left|1+d(S t, t)+d\left(S t^{\prime}, t^{\prime}\right)\right|} \\
& \leq\left(a_{1}+a_{7}+a_{9}+a_{12}\right) s^{2}|d(S t, t)|+\left(a_{2}+a_{8}+a_{10}\right) s^{2}\left|d\left(S t^{\prime}, t^{\prime}\right)\right| .
\end{aligned}
$$

Thus $|d(S t, t)| \leq \frac{a_{2}+a_{8}+a_{10}}{1-a_{1}-a_{7}-a_{9}-a_{12}}\left|d\left(S t^{\prime}, t^{\prime}\right)\right|$.

Similarly, we can show that $\left|d\left(S t^{\prime}, t^{\prime}\right)\right| \leq \frac{a_{2}+a_{8}+a_{10}}{1-a_{1}-a_{7}-a_{9}-a_{12}}|d(S t, t)|$.

Hence $S t=t$ and $S t^{\prime}=t^{\prime}$.Thus from (2),

$F\left(t, t^{\prime}\right)=S t=t$ and $F\left(t^{\prime}, t\right)=S t^{\prime}=t^{\prime}$

Similarly, consider

$$
\begin{aligned}
& s^{2}|d(t, T t)|=s^{3} \frac{1}{s}\left|d\left(t, G\left(t, t^{\prime}\right)\right)\right| \leq s^{3} \lim _{n \rightarrow \infty}\left|d\left(F\left(x_{n}, y_{n}\right), G\left(t, t^{\prime}\right)\right)\right| \\
& \leq \lim _{n \rightarrow \infty}\left[\begin{array}{l}
a_{1}\left|d\left(S x_{n}, T t\right)\right|+a_{2}\left|d\left(S y_{n}, T t^{\prime}\right)\right|+a_{3}\left|d\left(S x_{n}, F\left(x_{n}, y_{n}\right)\right)\right|+a_{4}\left|d\left(S y_{n}, F\left(y_{n}, x_{n}\right)\right)\right| \\
+a_{5}\left|d\left(T t, G\left(t, t^{\prime}\right)\right)\right|+a_{6}\left|d\left(T t^{\prime}, G\left(t^{\prime}, t\right)\right)\right|+a_{7}\left|d\left(S x_{n}, G\left(t, t^{\prime}\right)\right)\right| \\
+a_{8}\left|d\left(S y_{n}, G\left(t^{\prime}, t\right)\right)\right|+a_{9}\left|d\left(T t, F\left(x_{n}, y_{n}\right)\right)\right|+a_{10}\left|d\left(T t^{\prime}, F\left(y_{n}, x_{n}\right)\right)\right|+ \\
a_{11} \frac{\left|d\left(S x_{n}, F\left(x_{n}, y_{n}\right)\right)\right|\left|d\left(T t, G\left(t, t^{\prime}\right)\right)\right|}{\left|1+s d\left(S x_{n}, T t\right)+s d\left(S y_{n}, T t^{\prime}\right)\right|}+a_{12} \frac{\left|d\left(S x_{n}, G\left(t, t^{\prime}\right)\right)\right|\left|d\left(T t, F\left(x_{n}, y_{n}\right)\right)\right|}{\left|1+s d\left(S x_{n}, T t\right)+s d\left(S y_{n}, T t^{\prime}\right)\right|}
\end{array}\right] \\
& \leq a_{1} s|d(t, T t)|+a_{2} s\left|d\left(t^{\prime}, T t^{\prime}\right)\right|+a_{7} s|d(t, T t)|+a_{8} s\left|d\left(t^{\prime}, T t^{\prime}\right)\right|+a_{9} s|d(t, T t)| \\
& \quad+a_{10} s\left|d\left(t^{\prime}, T t^{\prime}\right)\right|+a_{12} \frac{s|d(t, T t)| s|d(t, T t)|}{\left|1+d(t, T t)+d\left(t^{\prime}, T t^{\prime}\right)\right|}
\end{aligned}
$$

Thus $|d(t, T t)| \leq \frac{a_{2}+a_{8}+a_{10}}{1-a_{1}-a_{7}-a_{9}-a_{12}}\left|d\left(t^{\prime}, T t^{\prime}\right)\right|$.

Similarly, we can show that $\left|d\left(t^{\prime}, T t^{\prime}\right)\right| \leq \frac{a_{2}+a_{8}+a_{10}}{1-a_{1}-a_{7}-a_{9}-a_{12}}|d(t, T t)|$.

Hence $T t=t$ and $T t^{\prime}=t^{\prime}$.Thus from $(3)$,

$G\left(t, t^{\prime}\right)=T t=t$ and $G\left(t^{\prime}, t\right)=T t^{\prime}=t^{\prime} \ldots(6)$

From (5) and (6), it follows that $\left(t, t^{\prime}\right)$ is a common coupled fixed point of $F, G, S$ and $T$. Uniqueness of common coupled fixed point follows easily from (2.1.1). Similarly, we can prove the theorem whenever $(2.1 .3)$ (b) holds.

Now we give an example to illustrate Theorem 2.1

Example 2.2 Let $X=[0,1]$ and $d(x, y)=i|x-y|^{2}, \forall x, y \in X$. Then $s=2$.

Define $F, G: X \times X \rightarrow X$ by $F(x, y)=\frac{x^{2}+y^{2}}{12}$ and $G(x, y)=\frac{x+y}{24}$ and $S, T: X \rightarrow X$ by $S x=x^{2}$ and $T x=\frac{x}{2}$.

Then $F(X \times X)=\left[0, \frac{1}{6}\right] \subseteq\left[0, \frac{1}{2}\right]=T(X)$ and $T(X)$ is a closed subspace of $X$.

Let $x_{n}=\frac{1}{n}, y_{n}=\frac{1}{\sqrt{n}} \in X$.Then

$\lim _{n \rightarrow \infty} F\left(x_{n}, y_{n}\right)=\lim _{n \rightarrow \infty} S x_{n}=\lim _{n \rightarrow \infty} F\left(y_{n}, x_{n}\right)=\lim _{n \rightarrow \infty} S y_{n}=0$. Thus $(2.1 .3)(a)$ holds. Clearly the pairs $(F, S)$ and $(G, T)$ are w-compatible. Now consider

$$
\begin{aligned}
& s^{3} d(F(x, y), G(u, v)) \\
& =8 i\left|\frac{x^{2}+y^{2}}{12}-\frac{u+v}{24}\right|^{2} \\
& =\frac{8}{576} i\left|2 x^{2}-u+2 y^{2}-v\right|^{2} \\
& \leq \frac{1}{72} 2 i\left[\left|2 x^{2}-u\right|^{2}+\left|2 y^{2}-v\right|^{2}\right] \\
& =\frac{i}{9}\left[\left|x^{2}-\frac{u}{2}\right|^{2}+\left|y^{2}-\frac{v}{2}\right|^{2}\right] \\
& =\frac{1}{9}[d(S x, T u)+d(S y, T v)]
\end{aligned}
$$


Thus (2.1.1) is satisfied with $a_{1}=a_{2}=\frac{1}{9}$ and $a_{i}=0$ for $i=3,4, \ldots, 12$. Thus all the conditions of Theorem 2.1 are satisfied and $(0,0)$ is the unique common coupled fixed point of $F, G, S$ and $T$.

If $s=1$ in Theorem 2.1, we have the following corollary.

Corollary 2.3 . Let $(X, d)$ be a complex valued metric space. Let $F, G: X \times X \rightarrow X$ and $S, T: X \rightarrow X$ be mappings satisfying the following :

$$
\begin{aligned}
& d(F(x, y), G(u, v)) \\
& \precsim a_{1} d(S x, T u)+a_{2} d(S y, T v)+a_{3} d(S x, F(x, y)) \\
& \quad+a_{4} d(S y, F(y, x))+a_{5} d(T u, G(u, v))+a_{6} d(T v, G(v, u)) \\
& \quad+a_{7} d(S x, G(u, v))+a_{8} d(S y, G(v, u))+a_{9} d(T u, F(x, y))+a_{10} d(T v, F(y, x)) \\
& \quad+a_{11} \frac{d(S x, F(x, y)) d(T u, G(u, v))}{1+d(S x, T u)+d(S y, T v)}+a_{12} \frac{d(T u, F(x, y)) d(S x, G(u, v))}{1+d(S x, T u)+d(S y, T v)}
\end{aligned}
$$

for all $x, y, u, v \in X$, where $a_{i}, i=1,2, \ldots ., 12$ are non-negative real numbers such that $\sum_{i=1}^{12} a_{i}<1$.

(2.3.2) The pairs $(F, S)$ and $(G, T)$ are $w$-compatible.

(2.3.3)(a) The pair $(F, S)$ satisfies property(E.A.), $F(X \times X) \subseteq T(X)$ and $S(X)$ is a closed sub space of $X$.

(or)

(2.3.3)(b) The pair $(G, T)$ satisfies property(E.A.), $G(X \times X) \subseteq S(X)$ and $T(X)$ is a closed sub space of $X$.

Then $F, G, S$ and $T$ have a unique common coupled fixed point.

\section{References}

1. A.Azam,B.Fisher and M.Khan,Common fixed point theorems in complex valued metric spaces, Numer. Funct. Anal. Optim. 32(3), (2011), 243-253.

2. C.Klin-eam and C.Suanoom,Some common fixed point theorems for generralized contractive type mappings on complex valued metric spaces, Abstr.Appl.Anal.,2013,6 pages,Article ID 604215, doi.10.1155/2013/604215.

3. F. Rouzkard and M. Imdad, Some common fixed point theorems on complex valued metric spaces, Comp. Math.Appls., 64,(2012), 1866-1874.

4. H. K. Nashine, M. Imdad and M. Hasan,Common fixed point theorems under rational contractions in complex valued metric spaces,J. Nonlinear Sci. Appl.,7,(2014), 42-50.

5. J.Kumar and S.Vashistha, Coupled fixed point theorem for generalized contraction in complex valued metric spaces,International Journal of Computer Applications,83(7),(2013),36-40.

6. K.Sitthikul and S.Saejung,Some fixed points in complex valued metric spaces, Fixed point theory Appl.,2012,11 pages,Article ID 189 .

7. K.P.R.Rao,P.Ranga Swamy and J.Rajendra Prasad,A common fixed point theorem in complex valued b-metric spaces,Bulletin of Mathematics and Statistics Research,1(1),(2013),1-8.

8. M.Abbas, M.Ali Khan and S.Radenovic, Common coupled fixed point theorems in cone metric spaces for w-compatible mappings, Appl.Math. Comput, 217, (2010), 195-202.

9. M.Abbas,B.Fisher and T.Nazir, Well-posedness and peroidic point property of mappings satisfying a rational inequality in an ordrerd complex valued-metric spaces,Sci. Stud. Res., Ser. Math. Inform. 22(1),(2012),5-24.

10. M.Abbas,M.Arshad and A.Azam,Fixed points of asympotically regular mappings in complex valued metric spaces, Georgian Math.J., 20, (2013), 213-221(2013):10.1515/gmj-2013-0013.

11. M. Aamri, D.El. Moutawakil,Some new common fixed point theorems under strict contractive conditions, J. Math. Anal. Appl., 27(1), (2002), 181Ü188.

12. M.A.Kutbi,A.Azam,J.Ahmad, and C.Di Bari, Some common coupled fixed point results for generalized contraction in complex-valued metric spaces,J.Appl.Math.,2013,10 pages,ArticleID 352927.

13. M.Kumar,P. Kumar and S. Kumar,Common fixed point theorems in complex valued metric spaces,J. Ana. Num. Theor. 2(2),(2014), 103-109 .

14. N.Malhotra and B.Bansal,Some common coupled fixed point theorems for generalized contraction in b-metric spaces, J. Nonlinear Sci. Appl., 8, (2015), 8-16.

15. N.Singh, D.Singh, A.Badal and V.Joshi, Fixed point theorems in complex valued metric spaces, Journal of the Egyptian Mathematical Society, (2015), 1-8 (In Press). 
16. R.K.Verma and H.K.Pathak,Common fixed point theorems for a pair of mappings in complex valued metric spaces,Journal of Mathematics and Computer Science, 6 (2013), 18-26.

17. R.K.Verma and H.K.Pathak, Common fixed point theorems using property (E.A) in complex-valued metric spaces, Thai Journal of Mathematics,11(2),(2013),347Ü355.

18. S.Chandok and D.Kumar,Some common fixed point results for rational type contraction mappings in complex valued metric spaces,Journal of Operators, 2013, 6 pages, Article ID 813707.

19. S.M. Kang, Coupled fixed point theorems in complex valued metric spaces, Int. Journal of Math. Analysis,7(46), (2013), 2269 - 2277.

20. T.G. Bhaskar and V. Lakshmikantham, Fixed point theorems in partially ordered metric spaces and applications, Nonlinear Analysis, 65(7),(2006), 1379 - 1393.

21. T.Mitra ,A common coupled fixed point result in complex valued metric space for two mappings,International Journal of Current Research, 7(8),(2015),19555-19559.

22. T.Mitra, A common coupled fixed point theorem in complex valued metric space for two mappings satisfying a rational inequality, International Journal of Scientific Research Engineering and Technology, 4(8), (2015), 831-835.

23. T.Senthil Kumar and R.Jahir Hussain,Common coupled fixed point theorem for contractive type mappings in closed ball of complex valued metric spaces,Adv.Inequal.Appl.2014,1-7,2014:34.

24. V. Lakshmikantham and Lj. Ciric, Coupled fixed point theorems for nonlinear contractions in partially ordered metric spaces, Nonlinear Analysis: Theory, Method. Appl., 70(12),(2009), 4341-4349.

25. W.Sintunavarat and P.Kumam, Generalized common fixed point theorems in complex valued metric spaces and applications, J. Inequal. appl., 2012, 11 pages,Article ID 84. 